\title{
Metastasis of Adrenocortical Carcinoma to Pericardial Fluid, Cytological Diagnosis (IHC) - A Case Report
}

\author{
Vasudha Nassa, Nandini N. M., Sushma K. L., Sapna Patel, Manjunath G. V. \\ Department of Pathology, JSS Medical College, Constituent College of JSS University, Mysore,Karnataka, India
}

Email Address:

nassa.vasudha@gmail.com (V. Nassa)

\section{To cite this article:}

Vasudha Nassa, Nandini N. M., Sushma K. L., Sapna Patel, Manjunath G. V.. Metastasis of Adrenocortical Carcinoma to Pericardial Fluid, Cytological Diagnosis (IHC) - A Case Report. Science Journal of Clinical Medicine. Special Issue: Latest Different Concepts of Gynaecology. Vol. 4, No. 4-1, 2015, pp. 31-35. doi: 10.11648/j.sjcm.s.2015040401.18

\begin{abstract}
A 54 years male patient presenting with multiple complaints was found to have large pericardial effusion leading to tamponade. Radiological findings were suggestive of a right adrenal mass. Cytological evaluation of the pericardial fluid revealed features of malignant effusion. Adrenocortical origin of the malignant cells was confirmed by immunostaining with A103 antibody against Melan-A. Hence, identification of primary lesion and confirmation of diagnosis was achieved by cytology with the help of immunohistochemistry performed on the cell block obtained from the drained pericardial fluid.
\end{abstract}

Keywords: Melan-A, Adrenocortical Carcinoma, Malignant Pericardial effusion, Cytology, Immunohistochemistry

\section{Introduction}

Adrenocortical carcinoma is a rare and highly malignant neoplasm with estimated annual incidence of 1.5 to 2 per million population. ${ }^{1}$ It grows rapidly and tends to metastasize to the liver, lungs and kidney. The prognosis is poor with a significant proportion [21 - 39\%] of patients having distant metastasis at the time of presentation. ${ }^{2}$

Cardiac metastasis of adrenocortical carcinoma is not unseen but is rare. ${ }^{3}$

Fluid cytology is considered to be the initial diagnostic approach in patients with effusions. The Cell Block technique can be considered as a useful adjuvant in evaluating the fluid for a final cytodiagnosis, along with the routine conventional smear method.

Antibody A103 against Melan A has potential value as a marker of Adrenocortical Carcinoma and can be used in the differential diagnosis. ${ }^{4}$

\section{Case Report}

A 54 years old male patient presented with epigastric pain, vomiting and accelerated hypertension and on evaluation contrast enhanced CT scan revealed adrenal haemorrhage (Figure 1). 2 D Echocardiography was suggestive of left sided pericardial effusion. The patient was stabilised and discharged on advice to follow up with USG abdomen every
15 days. On follow up USG, the adrenal lesion was found to shrink minimally in size.

On a later date, the patient presented with CVA and few days later, was admitted again with complaints of fever, jaundice, malaise. On evaluation, radiology revealed right adrenal mass with periportal, periaortic and aortocaval lymphadenopathy, mild hepatomegaly with fatty liver and cardiac tamponade with bilateral pleural effusion and minimal ascites. The pericardial fluid was tapped and sent for cytology which revealed features of malignant effusion.

The conventional smear from the fluid was satisfactory and showed clusters of pleomorphic tumor cells arranged in microacinar pattern. Individual cells showed abundant cytoplasm with altered nucleo-cytoplasmic ratio and hyperchromatic nuclei in the background of haemorrhage (Figure 2).

The section from the cell block showed sheets of pleomorphic tumor cells with individual cells showing abundant eosinophilic cytoplasm, hyperchromatic nuclei in a background of blood clot (Figure 3).

Lab reports were suggestive of anaemia, thrombocytopenia, hepatic dysfunction and increased levels of Vanillyl Mandelic Acid.

IHC staining with A103 antibody against Melan A was done on cell block obtained from the pericardial fluid which showed good number of cells with intracytoplasmic granular deposits favouring Melan-A positive cells ( Figure 4. ) confirming adrenocortical origin of the malignant cells. 
Hence, diagnosis of adrenocortical carcinoma with metastasis to pericardial fluid was made.

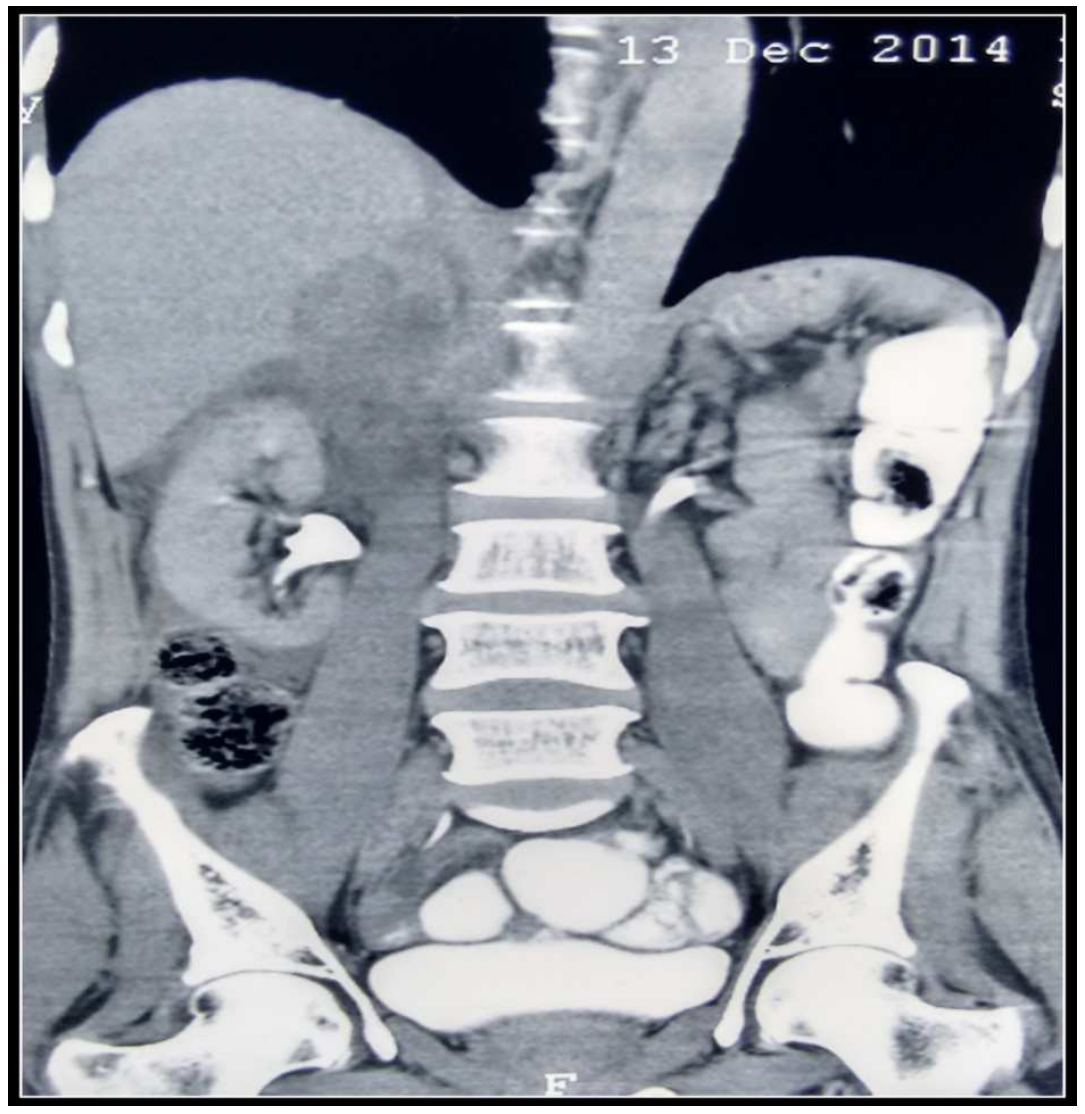

Figure 1. Contrast enhanced CT showing an ill-defined heterodense right suprarenal lesion measuring $4.9 \mathrm{~cm} \times 4.2 \mathrm{~cm}$.

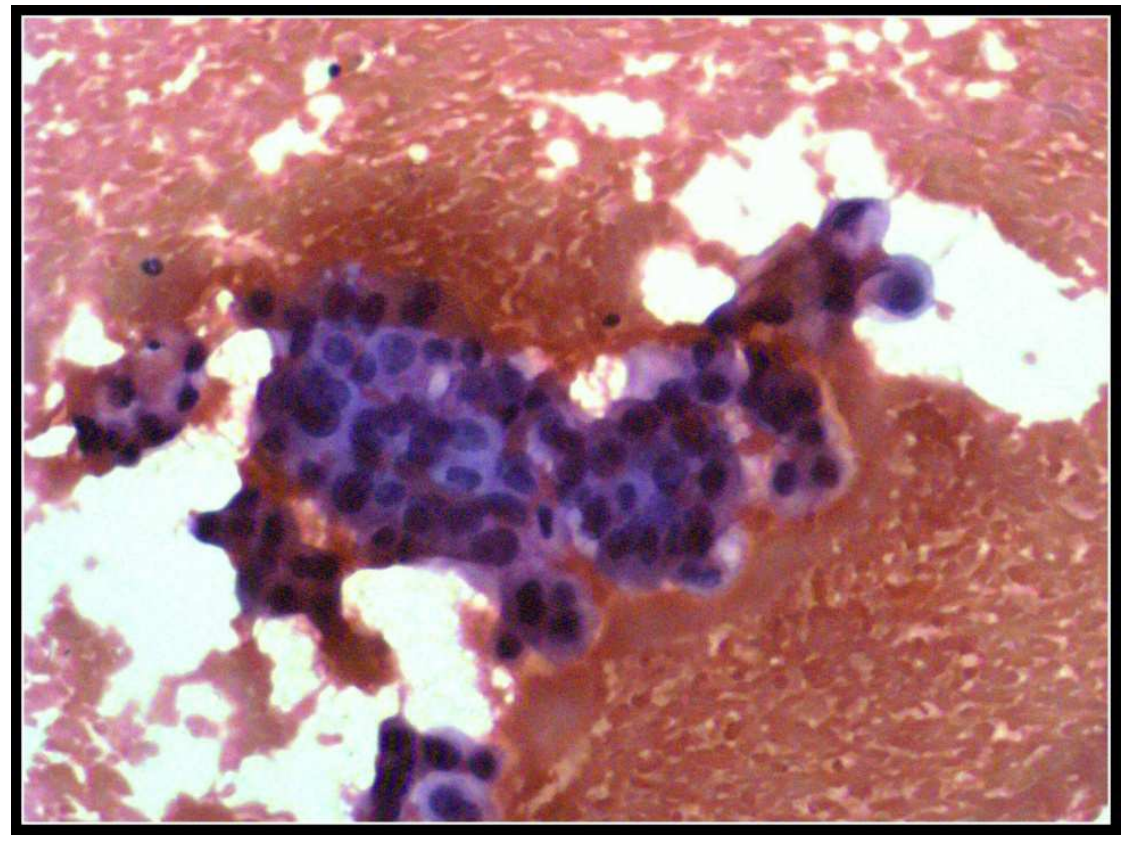

Figure 2. Conventional Smear showing pleomorphic tumor cells in acinar and microacinar pattern.( $H \& E, 40 x)$. 


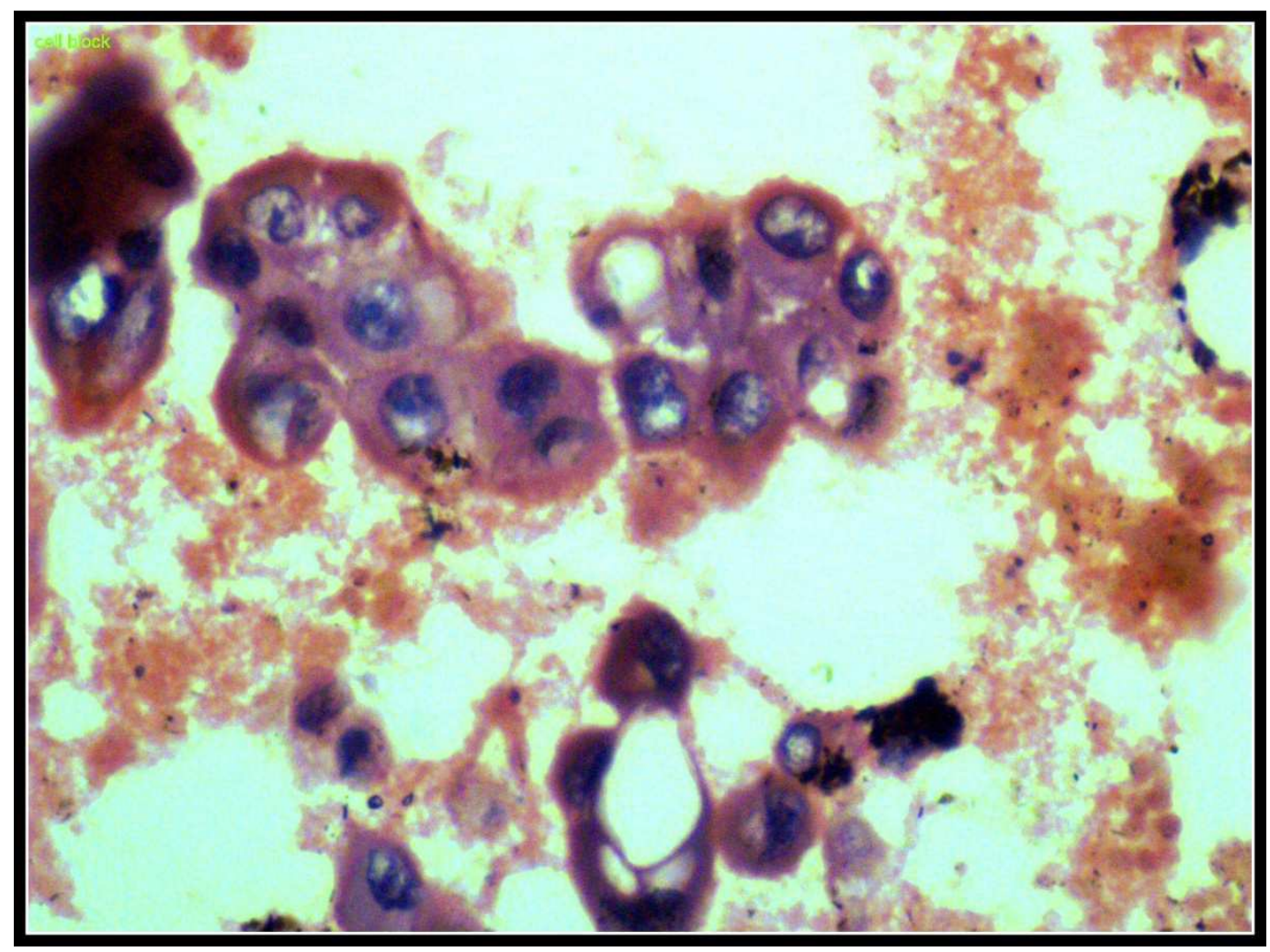

Figure 3. Section from Cell Block showing pleomorphic tumor cells with abundant cytoplasm and hyperchromatic nuclei.( H\&E, 40x).

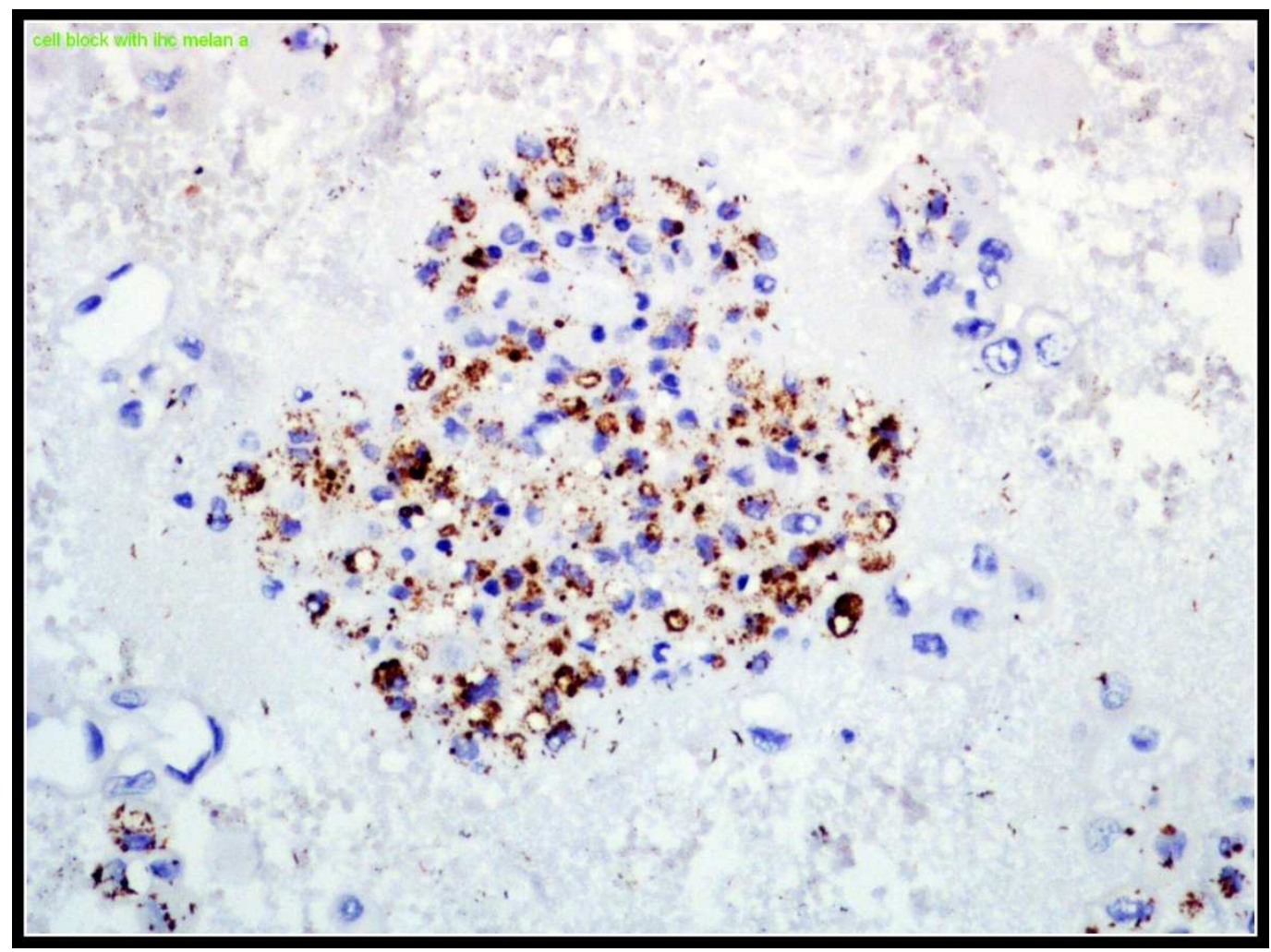

Figure 4. Section showing Immunoreactivity with Melan-A(A103)(40x).

\section{Discussion}

Carcinoma of the adrenal cortex is a rare but aggressive malignancy, accounting for 0.05 to $0.2 \%$ of all cancers, and
$0.2 \%$ of deaths caused by cancer ${ }^{5}$. The neoplasm arises in women twice as often as men, usually in the third to fifth decade of life. Another peak incidence is noted in children less than 6 years of age and girls are affected twice as 
common as boys. ${ }^{6}$ The right and the left adrenals are affected with equal frequency. Approximately $50 \%$ of adrenal cortical carcinomas are functioning and produce hormonal and metabolic syndromes leading to their discovery. The other $50 \%$ are silent and discovered only when they attain large size and produce localised abdominal symptoms or metastasis. ${ }^{7}$ The tumor grows rapidly and tends to metastasize to the liver and the lungs and invades the kidney, renal veins and inferior venacava. Dissemination occurs in $82 \%$ of patients with a median survival of 14.5 months. $^{8}$

Cardiac metastasis of adrenal carcinoma is rare. ${ }^{9}$ Malignant tumors which commonly metastasize to the heart include carcinoma lung, carcinoma breast, malignant lymphoma, leukemia, hypernephroma. The pericardium is the most common site of cardiac metastasis followed in frequency by the myocardium. Pericardial effusion is frequently an accompanying occurrence of pericardial metastasis. If it is advanced, cardiac tamponade ensues, requiring drainage.

The cytological examination of serous fluids is important in the diagnosis, staging and the prognosis of malignant lesions ${ }^{10}$. In some studies Fluid cytology study had a sensitivity of $92 \%$ and specificity of $100 \%$ for malignant effusion. ${ }^{11}$ The cytodiagnosis which is made by conventional smears has got a lower sensitivity due to the over crowding of the cells, cell loss and also due to the different laboratory processing methods. ${ }^{10}$

The Cell Block method yielded more cellularity with better architectural patterns and it improved the cytodiagnosis of malignancies. Hence, the Cell Block technique can be recommended as a useful adjuvant in evaluating the fluid cytology for a final cytodiagnosis, along with the routine Conventional Smear method. ${ }^{10}$

Melan A is a melanocyte differentiation antigen, recognised by autologous cytotoxic $\mathrm{T}$ lymphocytes. The MelanA/MART1gene encodes this protein, $2022 \mathrm{kDa}$, associated with endoplasmic reticulum and melanosomes. The function of the protein is unknown. Melan A is expressed in all normal melanocytes and melanocyte cell lines. Using the monoclonal antibody A103, staining is also seen in steroid hormone producing cells such as the adrenal cortex.

In a study conducted by Zhang et. al. anti Melan A was found to have $89 \%$ Immunoreactivity in case of Adrenal cortical tumors. $^{12}$

In another study conducted by Ghorab et. al. thirty two adrenocortical neoplasms, 86 renal cell carcinomas, and 57 hepatocellular carcinomas were evaluated by immunohistochemistry using the monoclonal antibody A103 The adrenocortical neoplasms were 21 adenomas and 11 carcinomas. 31 of the 32 adrenocortical neoplasms showed strong and diffuse granular cytoplasmic staining for Melan A. No nuclear reaction was observed. There were no differences in staining patterns between adrenocortical adenomas and carcinomas. With the exception of one clear cell renal cell carcinoma, all non-adrenocortical neoplasms were negative. The authors concluded that A103 is a useful immunohistochemical marker in the differential diagnosis of adrenocortical neoplasms from both renal cell and hepatocellular carcinomas. This marker, however, does not separate benign from malignant adrenocortical neoplasms. ${ }^{3}$

\section{Conclusion}

Fluid cytology is the initial diagnostic approach in patients with effusions and can help achieve the final confirmatory diagnosis with the help of adjuvant techniques including cell block and immunohistochemistry. In the present case differential diagnosis of a metastatic adrenocortical carcinoma was made based on the radiological and cytological findings of adrenal lesion, mediastinal lymphadenopathy and malignant pericardial effusion. Confirmatory diagnosis was made by immunostaining with A103 antibody against Melan A which is a potential marker of adrenocortical carcinoma and showed diffuse positivity in this case.

\section{References}

[1] Tauchmanova L, Colao A, Marzano LA, Sparano L, Camera L, Rossi A, Palmieri G, Marzano E, Salvatore M, Pettinato G, Lombardi G, Rossi R: Adrenocortical carcinomas: twelve-year prospective experience. World J Surg, 28:896-903,2004.

[2] Ng L, Libertino JM: Adrenocortical carcinoma: diagnosis, evaluation and treatment. J Urol , 169:5-11,2003.

[3] Nakata A, Yagi S, Oyama K, Kida H, Sugioka G: Adrenocortical carcinoma with a Giant Pericardial Mass. Internal Medicine,32:438 - 440, 1993.

[4] Ghorab, Zeina MD; Jorda, Merce MD, PhD; Ganjei, Parvin MD; Mehrdad MD: Melan A ( A103 ) Is Expressed in Adrenocortical Neoplasms but Not in Renal Cell and Hepatocellular Carcinomas. Applied immunohistochemistry and molecular morphology,11:330-333,2003.

[5] Brennan MF. Adrenocortical Carcinoma . CA Cancer J Clin,37:348-65,1987.

[6] Norton JA, Levin B, Jensen RT. Cancer of the endocrine system: the adrenal gland. DeVita VT Jr., Hellman S, Rosenberg SA (eds), Cancer: Principles and Practice of Oncology. Philadelphia, PA: JB Lippincott,1352-71,1993.

[7] Page DL, DeLellis RA, Hough AJ. Tumors of the adrenal (monograph) In: Atlas of Tumor Pathology, Washington D.C.: AFIP; 1986.

[8] Lutin JP, Cerdas S, Billiaud L, Thomas G, Gilhaume B, Bertagna X, Laudat MH, Louvel A, Chapuis Y and Blondeau P. Clinical features of adrenocortical carcinoma, prognostic factors, and the effect of mitotane therapy. N Engl J Med,322: 1195-1201,1990.

[9] Rosen B, Rozenman Y, Harpaz D: Extension of adrenocortical carcinoma into the right atrium - echocardiographic diagnosis: A case report. Cardiovascular Ultrasound, 1:5,2003.

[10] Shivakumar S. Udasimath; Surekha U. Arakeril; Mahesh H. Karigowdar; B. R. Yelikar: The Role of the Cell Block Method in the Diagnosis of Malignant Ascitic Fluid Effusions. JCDR:6(7)1280-1283,2012. 
[11] David G. Meyers; Rayma E. Meyers; Thomas W. Prendergast: The Usefulness of Diagnostic Tests on Pericardial Fluid. Chest,111(5):1213-1221,1997.

[12] Zhang P.J. ,M.D.; Gnerga E.M.,M.D.;Tomaszewski J.E. ,M.D.;
Pasha T.L.,B.S.; LiVolsi V.A., M.D. The Role oc Calretinin. Inhibin, Melan-A, BCL-2, and C-Kit in Differentiating Adrenal Cortical and Medullary Tumors: An

Immunohistochemical Study. Mod Pathol, 16(6):591597,2003 